\title{
xFitter 2.0.0: An Open Source QCD Fit Framework*
}

The xFitter Developers' Team: V. Bertone, ${ }^{a, b}$ M. Botje, D. Britzger, ${ }^{d}$ S. Camarda, A. Cooper-Sarkar, F. Giulif, A. Glazov, ${ }_{,}^{d}$ A. Luszczak, F. Olness ${ }_{,}^{h \dagger}$ R. Plačakytè, V. Radescu, ${ }^{e, f}$ W. Słomiński; ${ }^{j}$ and O. Zenaiev ${ }^{d}$

a Department of Physics and Astronomy, VU University, NL-1081 HV Amsterdam, The Netherlands

${ }^{b}$ Nikhef Theory Group Science Park 105, 1098 XG Amsterdam, The Netherlands

${ }^{c}$ Nikhef, Science Park, Amsterdam, The Netherlands

${ }^{d}$ DESY Hamburg, Notkestraße 85, D-22609, Hamburg, Germany

e CERN, CH-1211 Geneva 23, Switzerland

${ }^{f}$ University of Oxford, 1 Keble Road, Oxford OX1 3NP, United Kingdom

${ }^{g}$ T.Kosciuszko Cracow University of Technology, 30-084 Cracow, Poland

${ }^{h}$ SMU Physics, Box 0175 Dallas, TX 75275-0175, United States of America

${ }^{i}$ Institut für Theoretische Physik, Universität Hamburg, Luruper Chaussee 149, D-22761 Hamburg, Germany

${ }^{j}$ M. Smoluchowski Institute of Physics, Jagiellonian University, Lojasiewicza 11, 30-348 Kraków, Poland

xFitter [1] is an open-source package that provides a framework for the determination of the parton distribution functions (PDFs) of the proton for many different kinds of analyses in Quantum Chromodynamics (QCD). xFitter version 2.0.0 has recently been released, and offers an expanded set of tools and options. It incorporates experimental data from a wide range of experiments including fixed-target, Tevatron, HERA, and LHC. xFitter can analyze this data up to next-to-next-to-leading-order (NNLO) in perturbation theory with a variety of theoretical calculations including numerous methodological options for carrying out PDF fits and plotting tools which help visualize the results. While primarily based on the collinear factorization foundation, xFitter also provides facilities for fits of dipole models and transverse-momentum dependent (TMD) PDFs. The package can be used to study the impact of new precise measurements from hadron colliders, and also assess the impact of future colliders. This paper provides a brief overview of xFitter with emphasis of the new version 2.0.0 features.

XXV International Workshop on Deep-Inelastic Scattering and Related Subjects

3-7 April 2017

University of Birmingham, $U K$

*We would like to acknowledge Ringaile Plačakytė who served as convener of the HERA-Fitter/xFitter project from 2012 until March 2017, and to Voica Radescu who served as founder and convener from the start of the project in 2011 until June 2017. The outstanding dedication and service of these colleagues to this project was immeasurable and invaluable.

We acknowledge the hospitality of CERN, DESY, and Fermilab where a portion of this work was performed. This work was also partially supported by the U.S. Department of Energy under Grant No. DE-SC0010129. We are grateful to the DESY IT department for their support of the xFitter developers.

† Speaker. 


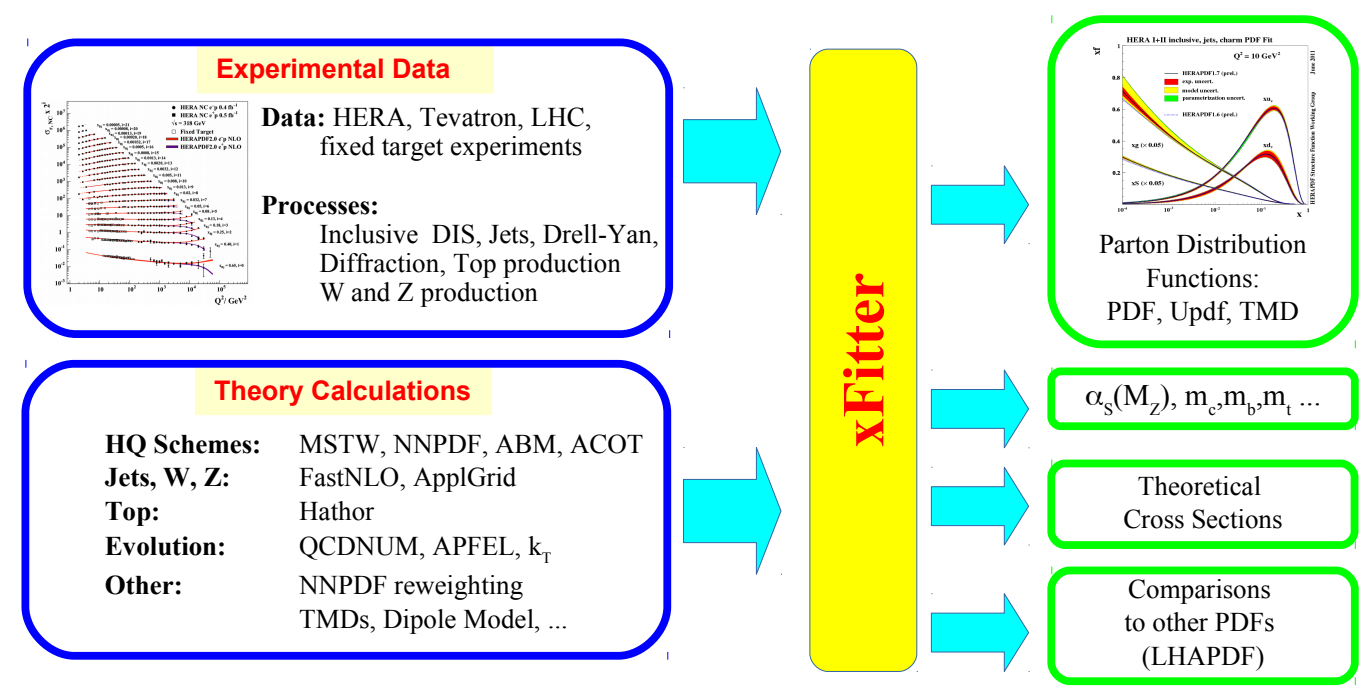

Figure 1: Schematic of the modular structure of xFitter illustrating the components and capabilities of the program.

\section{Introduction}

The Parton Distribution Functions (PDFs) are the essential components that allow us to make theoretical predictions for experimental measurements of protons and hadrons. The precision of the PDF analysis has advanced tremendously in recent years, and these studies are now performed with very high precision at NLO and NNLO in perturbation theory.

The xFitter project ${ }^{1}$ is an open source QCD fit framework that can perform PDF fits, assess the impact of new data, compare existing PDF sets, and perform a variety of other tasks [1]. The xFitter framework has already been used for more than 40 analyses including many LHC studies. ${ }^{2}$ The framework of xFitter is modular to allow for various theoretical and methodological options, and contains interfaces to QCDNUM [3],

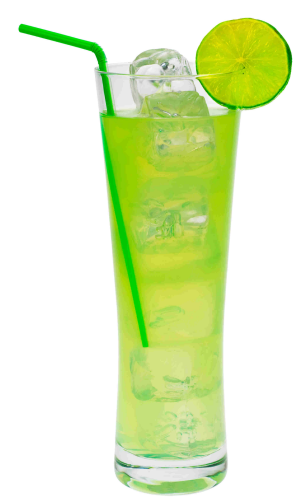

xFitter 2.0.0

(Frozen Frog)

iStock.com/Enjoylife2 APFEL [4], LHAPDF [5], APPLGRID [6], APFELGRID [7], FastNLO [8] HATHOR [9], among other packages. A schematic of the modular structure is illustrated in Fig. 1. xFitter also has a large number of data sets available, including a variety of fixed target experiments, HERA, Tevatron, and LHC. It is also possible to add new custom data sets such as LHeC and EIC pseudo-data.

The xFitter project grew out of PDF efforts of H1 and ZEUS which became the HERA-Fitter project in 2012, and renamed the xFitter project in 2015. xFitter is continually being updated, and version 2.0.0 (Frozen Frog) was released in March 2017 with many improvements and new features.

In this short report we will provide a brief tour of selected xFitter features with emphasis on some of the new additions; we also direct the reader to the appropriate literature where they can find more details. Additionally, we will discuss some of the xFitter tutorials available which can get the user up and running quickly with real analyses.

\footnotetext{
${ }^{1}$ xFitter can be downloaded from www.xFitter.org. An overview of the program can be found in Ref. [1].

${ }^{2} \mathrm{~A}$ complete list is available at www. xFitter.org.
} 


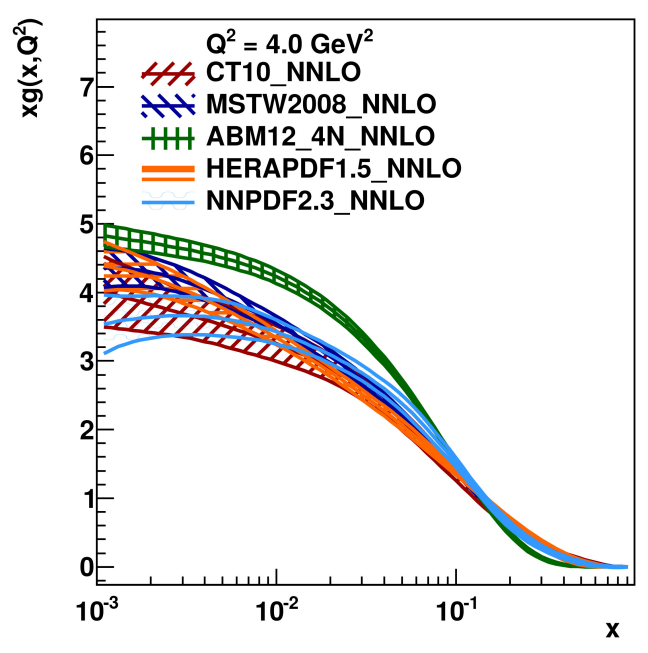

Figure 2: A selection of gluon PDFs with uncertainties at $Q^{2}=4.0 \mathrm{GeV}^{2}$, plotted using the drawing tools from $\mathrm{xFitter}$.

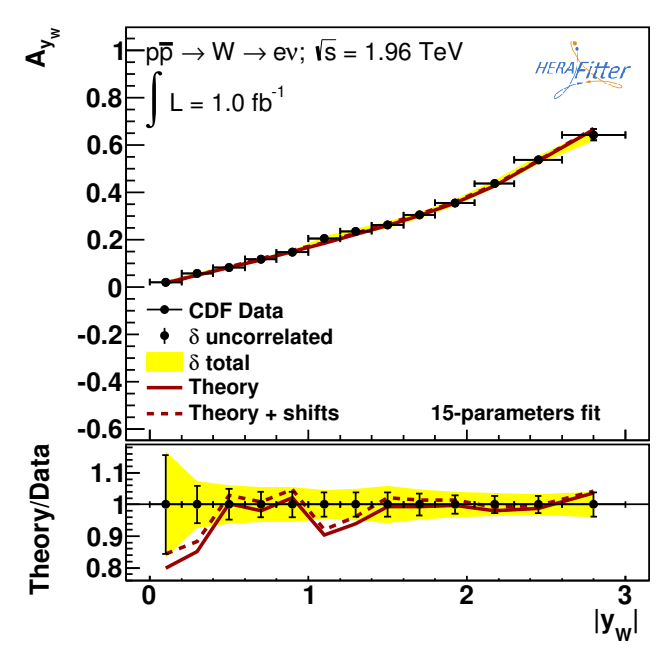

Figure 3: Comparison of theory predictions with Tevatron $W$ asymmetry with uncertainties [2].

\section{A Brief Tour}

The PDFs are the fundamental object that xFitter works with, and it has a variety of utilities to read, write, and manipulate the PDFs and associated uncertainties. For example, xFitter is able to read and write PDFs in the LHAPDF6 format [5], and Fig. 2 illustrates a sample plot of the PDFs from various global fitting projects.

xFitter can also generate comparison plots of data vs. theory, and an example is shown in Fig. 3. There are a variety of options for the definition of the $\chi^{2}$ function and the treatment of experimental uncertainties.

An important application of xFitter is to understand how a particular data set or experiment will impact the PDFs. In Fig. 4 we show the results using pseudo-data from a proposed $\mathrm{LHeC}$ and EIC experiment to constrain the relative uncertainty on the gluon distribution. ${ }^{3}$

$\mathrm{xFitter}$ is able to perform PDF profiling and reweighting studies. The reweighting method allows xFitter to update the probability distribution of a PDF uncertainty set (such as a set of NNPDF replicas) to reflect the influence of new data inputs. For the PDF profiling, xFitter compares data and MC predictions based on the $\chi^{2}$-minimization, and then constrains the individual PDF eigenvector sets taking into account the data uncertainties. We illustrate the profiling feature in Fig. 5 which displays the impact of a new data set (W/Z production in LHC lead collisions) on the nCTEQ15 PDFs [11]. In this case, the additional data set has reduced the PDF uncertainty of the original nCTEQ15 set. This example also illustrates that while xFitter cannot work with multiple nuclei simultaneously, it is able to work with a single nucleus (in this case lead); hence, xFitter can also be used for certain heavy ion analyses.

As many PDF analyses are now extended out to NNLO $\left[\mathrm{O}\left(\alpha_{S}^{2}\right)\right]$, the NLO QED effects $\left[\mathrm{O}\left(\alpha^{1}\right)\right]$

\footnotetext{
${ }^{3}$ The download of xFitter program includes a user manual and a sample HERA data set. Additional data sets are available at xfitter.hepforge. org including the LHeC pseudo-data set.
} 


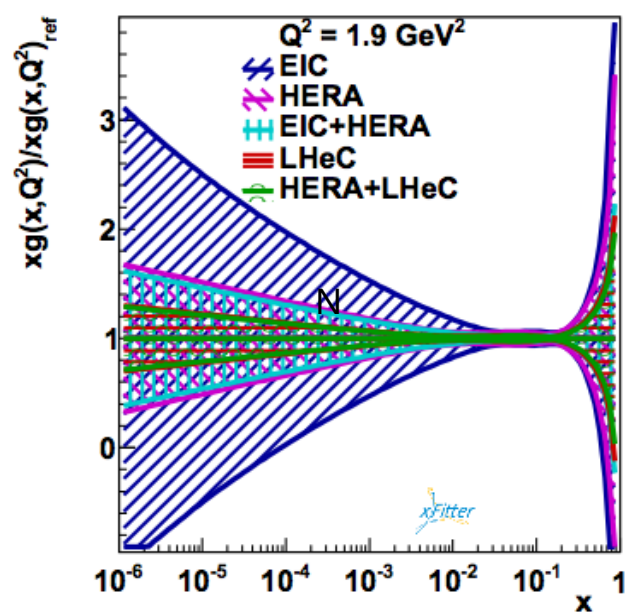

Figure 4: The PDF uncertainty as estimated by $\mathrm{xFitter}$ using pseudo-data from different experiments [10].

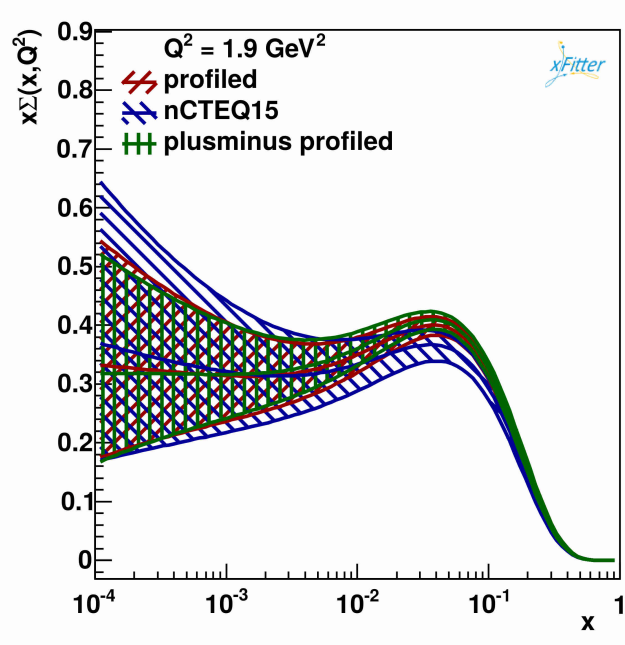

Figure 5: An example using xFitter profiling tools for nCTEQ15 lead nPDFs [11].

can also become important. ${ }^{4}$ For example, including QED processes in the parton evolution will break the isospin symmetry as the up and down quarks have different couplings to the photon. xFitter is able to include NLO QED effects, and this is illustrated in Fig. 6 which displays the photon PDF as determined using a NNLO QCD and NLO QED analysis [12].

Another new feature of $x$ Fitter is the ability to handle both pole masses and $\overline{M S}$ running masses in the FONNL scheme. While the pole mass is more closely connected to what is measured in experiments, the $\overline{M S}$ mass has advantages on the theoretical side of improved perturbative convergence. xFitter was used to perform a high precision determination of the $\overline{M S}$ charm mass in this new framework [13].

\section{Available Tutorials}

The best way to appreciate the capabilities of the xFitter program is to try the program with a variety of exercises. Here we provide a brief overview of some of the available tutorials. This range of applications should be sufficiently comprehensive to serve as a starting point for many types of xFitter analyses. ${ }^{5}$

Example 1: This is a basic PDF fit using the HERA I+II data just to become familiar with the separate $x$ Fitter inputs, outputs, and drawing tools. xFitter can compute at LO, NLO, and NNLO, and this exercise demonstrates a NNLO fit.

Example 2: This tutorial builds upon the previous exercise by using the HERA jets data to fit both the PDF and $\alpha_{S}$. In xFitter, $\alpha_{S}$ can either be fixed or floating, and we demonstrate this feature.

\footnotetext{
${ }^{4}$ See presentation by F. Giuli in these proceedings, "The photon PDF from high-mass Drell-Yan data at the LHC."

${ }^{5}$ The tutorials can be downloaded from the main xFitter website: www.xFitter.org. Examples 1-5 were designed by Stefano Camarda and presented at the "2016 CTEQ-MCnet School" at DESY, and Example 6 was designed by Voica Radescu and presented at the "Proton Structure in the LHC Era - School and Workshop."
} 


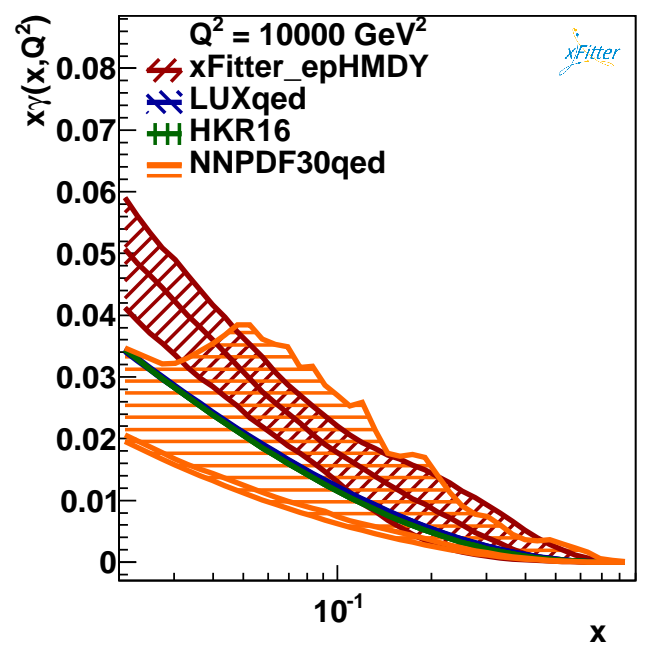

Figure 6: Comparison of the extracted photon $\mathrm{PDF} \quad x \gamma\left(x, Q^{2}\right)$ with other determinations from the literature [12].

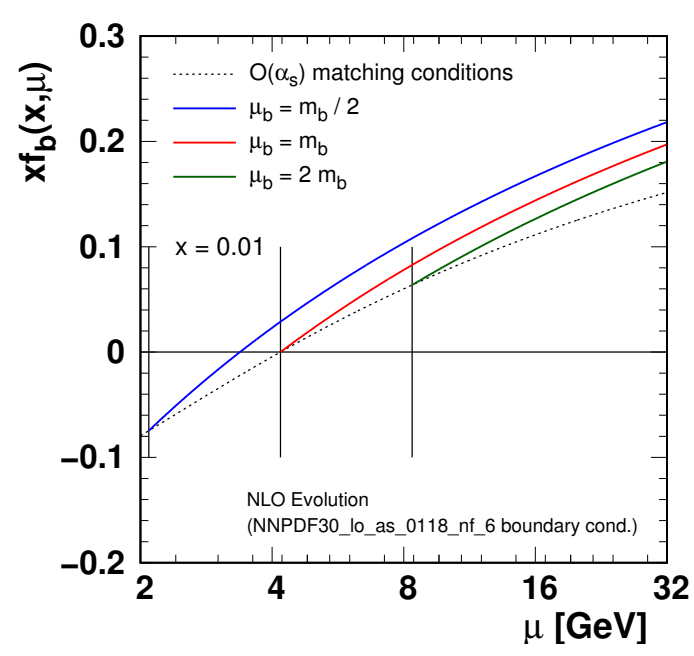

Figure 7: The $b$-quark PDF $x f_{b}(x, \mu)$ for different choices of the matching scales $\mu_{b}=$ $\left\{m_{b} / 2, m_{b}, 1 m_{b}\right\}$ as computed by xFitter in Ref. [14].

Additionally, the HERA jet data has uncertainty correlation matrices, and we illustrate how xFitter can work with a variety of statistical and systematic uncertainties.

Example 3: This exercise illustrates the profiling methodology. Starting with a given PDF in LHAPDF format, we add the Tevatron $\mathrm{W}$ asymmetry data set (with correlations) to determine the impact on the initial PDF. xFitter will then output the modified PDF set in to a LHAPDF format which can be used for calculation or comparison.

Example 4: There are variety of tools which are useful to examine xFitter output. This exercise uses Python with a Jupyter notebook to read the LHAPDF output from the previous xFitter example, manipulate the results, and generate comparison plots. Additionally, other tools are also available such as the Mathematica based ManeParse program [15].

Example 5: xFitter has a variety of tools available for the $\chi^{2}$ definition and the treatment of experimental uncertainties. This exercise demonstrates the equivalence of using the covariance matrix representation and the nuisance parameters (correlated systematic uncertainties) by running parallel fits and comparing the output.

Example 6: The measurement of the strange to down-sea quark ratio $r_{s}=s(x) / \bar{d}(x)$ has generated significant interest in the literature [16] as the result was near the SU(3) limit $r_{s} \sim 1$. This measurement uses LHC $W / Z$ production data to extract the strange PDF. This analysis can be reproduced in xFitter to allow the user to vary the inputs and the fitting parameters to validate the stability of this result.

While the above examples provide only a brief glimpse of xFitter's capabilities, the breadth of these topics should enable the users to quickly gain the necessary experience to proceed to their individual project. 


\section{Conclusion}

The xFitter 2.0.0 program is a versatile, flexible, modular, and comprehensive tool that can facilitate analyses of the experimental data and theoretical calculations. xFitter has been used for a variety of analyses of fixed target, Tevatron, HERA, and LHC data. It is a valuable tool for bench-marking and understanding differences between PDF fits, and it can provide impact studies for possible future colliders such as the LHeC, EIC, and FCC. We encourage use of xFitter, and welcome new contributions from the community to ensure xFitter continues to incorporate the latest theoretical advances and precision experimental data.

\section{References}

[1] HERA-Fitter developers' team: S. Alekhin et al. HERA-Fitter. Eur. Phys. J., C75(7):304, 2015.

[2] HERA-Fitter developers' team: S. Camarda et al. QCD analysis of $W$ - and Z-boson production at Tevatron. Eur. Phys. J., C75(9):458, 2015.

[3] M. Botje. QCDNUM: Fast QCD Evolution and Convolution. Comput. Phys. Commun., 182:490-532, 2011.

[4] Valerio Bertone, Stefano Carrazza, and Juan Rojo. APFEL: A PDF Evolution Library with QED corrections. Comput. Phys. Commun., 185:1647-1668, 2014.

[5] Andy Buckley, James Ferrando, Stephen Lloyd, Karl Nordström, Ben Page, Martin Rüfenacht, Marek Schönherr, and Graeme Watt. LHAPDF6: parton density access in the LHC precision era. Eur. Phys. J., C75:132, 2015.

[6] Tancredi Carli, et al., A posteriori inclusion of parton density functions in NLO QCD final-state calculations at hadron colliders: The APPLGRID Project. Eur. Phys. J., C66:503-524, 2010.

[7] Valerio Bertone, Stefano Carrazza, and Nathan P. Hartland. APFELgrid: a high performance tool for parton density determinations. Comput. Phys. Commun., 212:205-209, 2017.

[8] D. Britzger et al. [fastNLO Collaboration], doi:10.3204/DESY-PROC-2012-02/165.

D. Britzger, G. S. Klaus Rabbertz, F. Stober and M. Wobisch, PoS DIS 2015, 055 (2015).

[9] M. Aliev, H. Lacker, U. Langenfeld, S. Moch, P. Uwer and M. Wiedermann, "HATHOR: HAdronic Top and Heavy quarks crOss section calculatoR," Comput. Phys. Commun. 182, 1034 (2011).

[10] LHeC Study Group: J. L. Abelleira Fernandez et al. A Large Hadron Electron Collider at CERN: Report on the Physics and Design Concepts for Machine and Detector. J. Phys., G39:075001, 2012.

[11] nCTEQ Collaboration: K. Kovarik et al. nCTEQ15 - Global analysis of nuclear parton distributions with uncertainties in the CTEQ framework. Phys. Rev., D93(8):085037, 2016.

[12] xFitter developers' team: F. Giuli et al. The photon PDF from high-mass Drell Yan data at the LHC. Eur. Phys. J., C77(6):400, 2017.

[13] xFitter developers' team: Valerio Bertone et al. A determination of $m_{c}\left(m_{c}\right)$ from HERA data using a matched heavy-flavor scheme. JHEP, 08:050, 2016.

[14] xFitter developers' team: Valerio Bertone et al. Impact of the heavy quark matching scales in PDF fits. (In preparation.), 2017.

[15] D. B. Clark, E. Godat, and F. I. Olness. ManeParse : A Mathematica reader for Parton Distribution Functions. Comput. Phys. Commun., 216:126-137, 2017.

[16] Georges Aad et al. Determination of the strange quark density of the proton from ATLAS measurements of the $W \rightarrow \ell v$ and $Z \rightarrow \ell \ell$ cross sections. Phys. Rev. Lett., 109:012001, 2012. 